\title{
Estado de las desigualdades de género en el mercado de trabajo de El Salvador
}

Edgar Lara López ${ }^{1}$

Recibido en Julio 2011, aprobado en Octubre 2011

\section{Resumen}

Este artículo es un análisis del mercado de trabajo desde una perspectiva de género, con el propósito de determinar las condiciones y oportunidades de mujeres y hombres como fuerza de trabajo y como agentes económicos.

Palabras clave:

Mercado de trabajo, género, desigualdades, trabajo decente, indicadores

\begin{abstract}
This article is an analysis of the job market from a gender perspective, and in order to determine the conditions and opportunities of women and men as labor force and as economic agents
\end{abstract}

Keywords:

Job market, gender, inequalities, decent job, Indicators

1. Profesor investigador, radicado, edgarl_lo@hotmail.com 


\section{Introducción}

A diferencia del mercado de bienes, en donde las fuerzas del mercado suelen determinar las condiciones de intercambio y los precios, el mercado de trabajo, es una institución social en donde si bien hay una interacción de la oferta y demanda, también interviene un conjunto de normas sociales que condicionan o facilitan el estilo de inserción de mujeres y hombres en la economía. Integra un marco regulatorio o legal que establece las condiciones mínimas de trabajo, formas de contratación y organización social de la población trabajadora.

Las repercusiones de las normas sociales y el marco regulatorio del mercado de trabajo no son neutrales en términos de género, lo cual se constata cuando se observa que mujeres y hombres se enfrentan a condiciones y oportunidades desiguales tanto en la esfera de trabajo remunerado como en el no remunerado. Asimismo, desde la perspectiva de género y de la economía feminista, este mercado constituye una de las áreas más importantes de estudio, investigación y teorización en la economía debido a que es la fuerza de trabajo humana la que se sitúa en el centro del debate, son evidentes las marcadas desigualdades entre mujeres y hombres en cuanto a condiciones de trabajo, estatus y roles que asumen en el mercado. Además, es un espacio propicio para la implementación de políticas que aseguran la igualdad de oportunidades y la erradicación de la pobreza.

En este sentido, este artículo tiene como objetivo contribuir al análisis y caracterización del mercado de trabajo salvadoreño desde una perspectiva de género a fin de determinar el estado de las desigualdades entre mujeres y hombres a la luz de la noción de trabajo decente y los compromisos en materia económica y de trabajo expuestas en las convenciones sobre el adelanto de las mujeres.

Para ello se ha organizado en dos apartados: el primero expone un marco conceptual de análisis del mercado de trabajo desde la perspectiva de género; el segundo, expone el estado de las desigualdades de género a través de un conjunto de indicadores en doce áreas del mercado de trabajo: pobreza de la fuerza de trabajo, participación en el trabajo no remunerado, oportunidades de empleo, el empleo precario, segregación ocupacional, ejercicio del poder y acceso a la propiedad de las empresas, salarios, educación o formación de la fuerza de trabajo, estabilidad laboral, seguridad social, trabajo infantil, y sindicalización.

Finalmente, se presentan las conclusiones y consideraciones finales que desarrollan los principales hallazgos, los cuales señalan que el mercado de trabajo salvadoreño se ve determinado por un orden de género que tiene su base en la organización patriarcal de la sociedad, y evidencia la existencia de 
desigualdades de género en la que las mujeres tienen una posición desventajosa con respecto a los hombres.

Este artículo ha sido elaborado en calidad de becario del programa de becas MAEC-AECID del Ministerio de Asuntos Exteriores y de Cooperación y la Agencia Española de Cooperación Internacional para el Desarrollo. Una versión más amplia y detallada del estudio ha sido publicada en el informe anual "Mujer y Mercado laboral 2010" de la Organización de Mujeres Salvadoreñas por la Paz -ORMUSA-.

\section{El mercado de trabajo desde una perspectiva de género}

\subsection{El orden de género y el mercado de trabajo}

Si partimos de la noción de género², que propone la historiadora Joan Scott, este se constituye cuando hay una interrelación entre los símbolos y los mitos culturalmente disponibles, la normatividad que da sentido a los mismos, las instituciones que los refuerzan, reproducen e intervienen y la identidad subjetiva de cada persona o grupo. En concordancia con dicha noción, el mercado de trabajo se constituye como una institución capaz de reproducir muchos de los roles y estereotipos de género que ubica a las mujeres y hombres en posiciones sociales distintas, con oportunidades y expectativas de desarrollo personal desigual.

Como se indica en la introducción, desde la perspectiva de género, el mercado de trabajo se ha considerado como una de los principales áreas de atención dentro de la economía; en primer lugar, porque es un mercado cuya principal mercancía de transacción es la fuerza de trabajo humana; en segundo lugar, porque es donde más se evidencian las desigualdades de género; y finalmente, porque constituye un eje de política económica estratégico para la consecución de una sociedad más equitativa.

El mercado de trabajo es una institución social en donde, además de la interacción entre la población trabajadora y empleadora, interviene un conjunto de normas sociales que condicionan o facilitan la inserción en el trabajo, así como también integra un marco regulatorio o legal que establece las condiciones mínimas de trabajo, formas de contratación y organización social de la oferta y demanda de la fuerza de trabajo.

En este sentido, en la configuración del mercado de trabajo influyen factores normativos tanto de orden social como legal e institucional. Específicamente

2. En el artículo titulado "Apuntes sobre la categoría género desde una lectura antropológica” publicado en Teoría y Praxis 17, julio 2010, se puede encontrar una explicación más amplia de la categoría género. 
se identifican los siguientes factores: económicos, políticos (institucionales y legales), demográficos y de orden de género; cada uno de ellos o en su conjunto pueden repercutir en una participación igualitaria o no de mujeres y hombres en el mercado de trabajo.

Como se observa en la Figura 1 la oferta y demanda de la fuerza de trabajo y su interacción en el mercado está determinada por los cuatro factores; así el entorno económico, el modo de producción, la disponibilidad de recursos, el grado de desarrollo empresarial y tecnológico condicionan el tipo de trabajo que se genera en la economía y el tipo de mano de obra necesaria para el desempeño del mismo. Por otra parte, el modelo de crecimiento económico que asumen los países y/o las prioridades sectoriales genera transformaciones en la oferta de trabajo a fin de que esta se pueda ajustar y responder a las nuevas necesidades y oportunidades que impone el modelo. De igual manera la demanda no está exenta ya que en ella se concretizan las prioridades productivas del modelo de crecimiento.

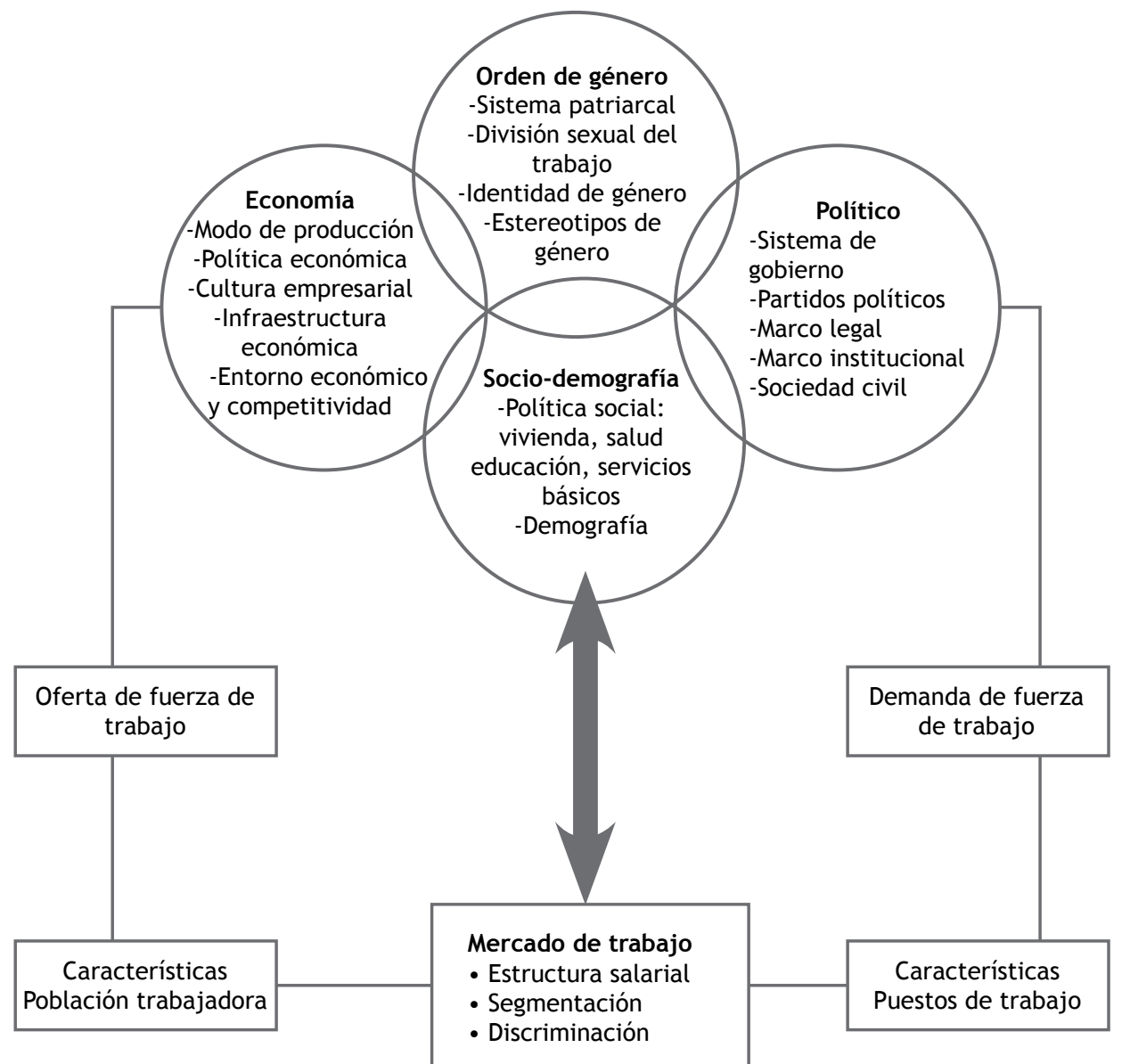

Fuente: Adaptado de Miedes, Blanca (2010). 
La política a través de la legislación laboral o regulación económica, el tipo de gobierno, los partidos políticos, el marco institucional gubernamental y las expresiones políticas de la sociedad civil como sindicatos, empresariado y promotores de derechos humanos impactan en el mercado de trabajo y en la caracterización de la demanda y la oferta. El tipo de régimen legal puede generar tipos de mercados de trabajo más flexibles o regidos, el marco institucional y su nivel de desarrollo genera confianza o desconfianza entre la población trabajadora en cuanto a la función de protección de los derechos laborales. La incidencia y cabildeo político de los sindicatos, asociaciones empresariales y organizaciones de derechos humanos repercute en el funcionamiento del mercado de trabajo en la medida que estos generan presiones para el mejoramiento de las condiciones laborales o para una gestión de la fuerza de trabajo que responda a las prioridades y necesidades del empresariado.

El factor socio-demográfico incide en gran medida sobre la oferta de trabajo; la política demográfica de un país puede incidir considerablemente en un exceso o déficit de la fuerza de trabajo, así como también la política educativa y de formación profesional indica el desarrollo y calidad de la población trabajadora. Aspectos de la política social como el acceso a la vivienda y servicios básicos son fundamentales para la productividad de las personas. Sin duda, la migración actualmente es un fenómeno que tiene su base en la necesidad de los hogares de salir de la pobreza o de mejorar las condiciones de vida de sus miembros; los movimientos migratorios afectan la estructura de los mercados de trabajo tanto de los países receptores como de los expulsores; así como también los movimientos internos del campo a la ciudad repercuten en la necesidad de una reestructuración productiva y consecuentemente de la demanda de la fuerza de trabajo.

En cuanto al orden de género, la sociedad y el sistema patriarcal tienen un conjunto de reglamentos o normas que regulan el género; también el género instituye su propio orden o régimen regulador y disciplinador que opera en la sociedad y condiciona a las personas, en su sexualidad e identidad propia. Las personas y los cuerpos que son producidos o adaptados a ese reglamento del género pueden estar condicionados, marginados y discriminados, y en términos del mercado de trabajo esto se traduce en una participación desigual entre hombres y mujeres; no obstante, el género tiene la capacidad de desconstruirse y rehacerse.

El orden de género imperante que tiene su base y reproduce el orden patriarcal y heteronormativo, condiciona considerablemente la fuerza de trabajo y la forma cómo se insertan las mujeres y los hombres en la economía, así como también en las percepciones del empresariado (demanda de trabajo) sobre el valor de la fuerza de trabajo masculina y femenina. 
Es reconocido que las mujeres se insertan en el mercado de trabajo en condiciones desiguales con respecto a los hombres, lo cual tiene su origen en la desvalorización de lo femenino y sobrevalorización de lo masculino presente en las sociedades de tipo patriarcal. Tal situación "coloca a hombres y mujeres en lugares sociales distintos y desiguales, con atribuciones, derechos y deberes distintos y desiguales; lugares construidos estructuralmente, más allá de las intenciones de la psique individual."3

Estas valorizaciones dan lugar al surgimiento de la división sexual, la cual asigna "tareas excluyentes y de distinto valor social según el sexo. Desde el orden social de género a los hombres y a las mujeres se les asignan dos espacios $o$ ámbitos excluyentes". Así, a las mujeres se le asignan todas las actividades de cuidado del hogar y la reproducción de la fuerza de trabajo, mientras que a los hombres se le asignan roles en lo público: lo político, lo económico y lo social.

Los cuatros factores expuestos anteriormente, no actúan de manera autónoma sobre el mercado de trabajo, no son independientes unos de otros, cada factor tiene la potencialidad de influir sobre otro, el económico se puede ver condicionado por el político, el demográfico responder al económico. Pero no cabe duda que estos tres son reproductores del orden de género. Por otra parte, las tensiones entre los actores del mercado de trabajo y las condiciones de trabajo de la población trabajadora pueden generar cambios en los factores a fin de que estos respondan a las necesidades del mercado.

\subsection{El trabajo reproductivo y/o doméstico}

Teniendo presente el orden de género, la división sexual del trabajo supone dos tipos de trabajo con valoraciones distintas. Por una parte está el trabajo productivo que se realiza en ámbito público, en el mercado, socialmente valorizado, remunerado y con mayor participación masculina. Por otra parte, el trabajo reproductivo que se realiza en el ámbito privado, en el hogar, no remunerado, desvalorizado y con mayor participación femenina.

Si bien la economía ha profundizado y teorizado en el trabajo remunerado, el abordaje del trabajo no remunerado y/o doméstico no ha sido suficientemente investigado, y cuando se ha hecho ha sido para justificar el orden de género. No obstante, las economistas feministas han realizado una labor importante en su análisis, valorización y teorización. Si bien para la economía clásica el trabajo en la esfera privada carece de valor, las mujeres dedicadas al trabajo del hogar no están inactivas, sino que están trabajando y constituye un trabajo importante para el sostenimiento de la vida y para el desempeño de la fuerza de trabajo, por lo que consecuentemente genera valor.

3. OIT-IPEC, explotación sexual comercial y masculinidad, P. 54

4. CEPAL-UNFPA, Masculinidad y factores socio culturales asociados al comportamiento de los hombres P. 127 
A partir de la división sexual del trabajo, se identifican dos tipos de trabajo: trabajo remunerado y trabajo no remunerado, que para los fines de este artículo, engloba las nociones de trabajo reproductivo o de reproducción social y doméstico. El trabajo no remunerado constituye la fuerza de trabajo invertida para la reproducción de la misma, el cuidado y el bienestar familiar; por lo general, es desempeñando en el ámbito del hogar o espacios vinculados a este que garanticen la convivencia comunitaria.

El trabajo no remunerado incluye la definición de trabajo doméstico, que se define como "el cuidado del mantenimiento de los espacios y bienes domésticos, así como el cuidado de los cuerpos, la educación, la formación, el mantenimiento de las relaciones sociales y el apoyo psicológico a los miembros de la familia"'. Por lo general, el trabajo no remunerado (y doméstico) supone una obligación que tiene su costo en términos de tiempo y energía, es un trabajo sin retribución monetaria y es indispensable para la sostenibilidad de la vida ${ }^{6}$.

Según Piccho, la importancia del trabajo doméstico y en consecuencia del trabajo no remunerado reside en que permite ampliar la renta monetaria, expandir el nivel de vida. Por otra parte, produce y prepara fuerza de trabajo, absorbe las tensiones de las personas en el trabajo remunerado, contribuye a la selección de personas y al mejoramiento de las capacidades de las mismas para el mercado. Esta economista a fin de visibilizar el aporte del trabajo no remunerado en la macroeconomía, lo introduce como un elemento clave en el flujo circular de la economía y para la eficiencia económica. Como lo indica, este es un trabajo que genera bienestar a los miembros del hogar, produce bienes y servicios necesarios para que la fuerza de trabajo sea más productiva, prepara y forma a la fuerza de trabajo y constituye un filtro de personas que salen al trabajo remunerado, en suma es un espacio de desarrollo humano. La autora plantea que al situarlo en una perspectiva macroeconómica, el trabajo no remunerado pierde su connotación familiar y femenina, se convierte un elemento clave para la eficiencia del sistema económico, por lo que el papel del Estado en las relación familia - empresa es fundamental ${ }^{7}$.

La introducción del trabajo no remunerado en el funcionamiento de la economía, permite entender de manera sencilla el rol que juega este en el desarrollo humano y su interconexión con la esfera de producción y distribución. Permite entender por qué las relaciones al interior de las familia no son armónicas; ayuda a comprender cómo las roles de género pueden condicionar el estilo de inserción laboral de los integrantes de la familia, así como también el salario que se percibe en la esfera mercantil.

5. A. Picchio, Un enfoque macroeconómico ampliado de las condiciones de vida, P. 2

6. M. A. Rivas, Desigualdades de género en el mercado laboral

7. A. Picchio, ibid 


\subsection{Trabajo decente y convenciones para el adelanto de las mujeres}

Desde la perspectiva internacional, El Salvador ha mostrado una actitud positiva en cuanto a adherirse o ratificar la mayoría de acuerdos o convenciones internacionales que promuevan la igualdad de oportunidades para hombres y mujeres.

Por una parte, ha ratificado la Convención sobre la Eliminación de todas las formas de Discriminación contra la Mujer -CEDAW por sus siglas en inglés-, mediante la cual el Estado salvadoreño, en materia económica y laboral, se ha comprometido a adoptar medidas para eliminar la discriminación contra la mujer, a asegurar condiciones de igualdad entre hombres y mujeres, velar por el derecho al trabajo, establecer unas mismas oportunidades de empleo, permitir el derecho al ascenso, garantizar la estabilidad, la formación, la igual remuneración, la seguridad y la protección social, garantizar la protección de la maternidad y generar acciones que faciliten a los padres la combinación de las obligaciones familiares con las responsabilidades del trabajo.

De igual manera, ha asumido la Declaración y Plataforma de Acción de Beijing de la Cuarta Conferencia Mundial sobre la Mujer, en la que el país se muestra decidido a fomentar la independencia económica de las mujeres, los derechos económicos de las mismas, el acceso de estas al control de los recursos, eliminar la segregación sexual y toda forma de discriminación, adoptar medidas orientadas a la superación de la pobreza de las mujeres y la armonización de las responsabilidades de mujeres y hombres en la familia y el trabajo.

Asimismo, se ha adherido a las aspiraciones de la Organización Internacional del Trabajo de promover acciones encaminadas a que las personas consigan un trabajo decente, es decir, un trabajo que se desarrolle en condiciones de libertad, equidad, seguridad y dignidad; la promoción del trabajo decente implica la generación de oportunidades de empleo e ingresos, respeto de los principios y derechos fundamentales del trabajo, la protección y seguridad social, y el diálogo social y tripartito.

\section{Estado de las desigualdades de género en el mercado de trabajo}

A partir de un conjunto de indicadores y teniendo presentes, la perspectiva de género, las consideraciones de la CEDAW, la plataforma de acción de Beijing, la noción de trabajo decente y la disponibilidad de información estadística se ha determinado el estado de las desigualdades de género en doce áreas del mercado de trabajo salvadoreño: (1) la situación de pobreza de la fuerza de trabajo, (2) la participación en el trabajo no remunerado, (3) oportunidades de empleo, (4) el empleo precario, (5) segregación ocupacional, (6) ejercicio del poder y acceso a la propiedad de las empresas; (7) salarios, (8) educación o 
formación de la fuerza de trabajo, (9) estabilidad laboral, (10) seguridad social, (11) trabajo infantil, y (12) sindicalización. El Cuadro 1, presenta los estados para cada una de las áreas.

\subsection{La situación de pobreza de la fuerza de trabajo}

La incidencia de la pobreza es levemente mayor en las mujeres: para el 2009 el $38.4 \%$ de los hogares liderados por mujeres estaban en pobreza y el $37.4 \%$ en el caso de los hogares con jefatura masculina. Es importante señalar que entre 2007 y 2008 la pobreza en los hogares se incrementó en 5.4 puntos porcentuales, pero en el 2009 se reduce en 2.1 puntos con respecto al 2008.

En cuanto a la fuerza de trabajo, el $38.8 \%$ de la fuerza de trabajo femenina (se refiere a la Población Económicamente Activa -PEA-) y el 37.4\% de la masculina están en pobreza. Adicionalmente el $\mathbf{4 7 . 5 \%}$ de la fuerza de trabajo femenina y el $31.5 \%$ de la masculina no reporta algún tipo de ingreso.

\section{2 la participación en el trabajo no remunerado}

El trabajo no remunerado constituye una de las áreas del mercado de trabajo con marcadas desigualdades de género; al 2009 la población en edad de trabajar en dicha actividad asciende a 831,389 personas, de las cuales el $97.9 \%$ son mujeres y el $2.1 \%$ son hombres. Dentro de la Población Económicamente Inactiva -PEI, las mujeres tienen una participación en el trabajo no remunerado (trabajo del hogar y responsabilidades familiares) del $69.9 \%$, en tanto que los hombres sólo participan con un $5.1 \%$. Estos indicadores confirman que la inserción de la personas al trabajo está condicionada por la división sexual del trabajo, las mujeres están más dedicadas al trabajo para el hogar o reproducción y los hombres al trabajo para el mercado.

Los indicadores señalan que las mujeres son las que más combinan el trabajo no remunerado con el remunerado o que posiblemente procuran conciliar la vida del hogar con la del trabajo remunerado; del total de mujeres ocupadas (dentro de la PEA) con una jornada laboral menor a 40 horas semanales, el 14.4\% expone como motivo de una menor jornada los quehaceres domésticos y razones familiares; ese motivo representa para los hombres el $1 \%$. Adicionalmente, para las mujeres en comparación con los hombres el trabajo no remunerado constituye una razón mayor para abandonar el trabajo en el mercado: el 8.6\% de la mujeres desocupadas exponen como motivo de su situación o de abandono de su trabajo el hecho que debía realizar tareas del hogar, porcentaje que para los hombres es del $0.7 \%$. 


\subsection{Oportunidades de empleo}

Los indicadores evidencian que existe un alto déficit en términos de género en cuanto a iguales oportunidades de empleo para hombres y mujeres; especialmente son las mujeres quienes tienen una menor participación en el empleo: sólo el $45.2 \%$ de las mujeres en edad de trabajar están empleadas (tasa de empleo), en tanto que la tasa de los hombres es del $73.7 \%$ para 2009, lo cual repercute en una mayor dependencia económica de las mujeres

\subsection{Empleo precario}

El empleo precario es contrario a los principios de seguridad y dignidad humana y de la noción de trabajo decente, ya que se trata de trabajos con alta vulnerabilidad, poca protección social, dependencia y bajos ingresos. Los indicadores que se aproximan a dicha situación es la tasa de subempleo y empleo informal.

Si bien El Salvador presenta una baja tasa de desempleo (7.3\%), su mayor problema reside en el subempleo y la informalidad en el empleo. Para 2009, el $34.5 \%$ de la población ocupada en el área urbana estaba en situación de subempleo y su incidencia es mayor en las mujeres, quienes tienen una tasa del $36.4 \%$, mientras que la de los hombres es del $33 \%$.

En términos de género se constata una feminización del empleo informal: más de la mitad de las mujeres (el 57.4\%) en el área urbana está en situación de informalidad en comparación con un $44.6 \%$ de hombres. Es decir, que para las mujeres este sector constituye una de las principales fuentes de empleo. 
Cuadro 1

\section{Indicadores del mercado de trabajo}

\begin{tabular}{|c|c|c|c|c|c|c|c|c|c|}
\hline \multirow{2}{*}{ Indicadores } & \multicolumn{3}{|c|}{ Pais } & \multicolumn{3}{|c|}{ Hombres } & \multicolumn{3}{|c|}{ Mujeres } \\
\hline & 2007 & 2008 & 2009 & 2007 & 2008 & 2009 & 2007 & 2008 & 2009 \\
\hline \multicolumn{10}{|l|}{ Pobreza en la fuerza de trabajo } \\
\hline Hogares en situación de pobreza (\$) & 34.6 & 39.9 & 37.8 & 34.4 & 39.7. & 37.4 & 35.0 & 40.4 & 38.4 \\
\hline PET en situación de pobreza (\$) & 34.9 & 40.9 & 38.1 & 34.2 & 40.3. & 37.3 & 35.4 & 41.4 & 38.8 \\
\hline PEA en situación de pobreza (\%) & 30.7 & 36.7 & 34.4 & 33.9 & 40.4 & 38.0 & 26.1 & 31.4 & 29.2 \\
\hline $\begin{array}{l}\text { Población ocupada en TNR en situación de pobreza } \\
\text { (\%) }\end{array}$ & 48.1 & 54.4 & 52.3 & 48.0 & 46.2 & 39.0 & 48.1 & 54.6 & 52.6 \\
\hline $\begin{array}{l}\text { Población (PET) que no reporta algún tipo de ingresos } \\
\text { (\%) }\end{array}$ & 36.8 & 36.1 & 40.2 & 23.1 & 22.2 & 31.5 & 47.9 & 47.7 & 47.5 \\
\hline \multicolumn{10}{|l|}{ Trabajo no remunerado } \\
\hline $\begin{array}{l}\text { Población (PEI) en quehaceres domésticos y } \\
\text { obligaciones familiares }(X)\end{array}$ & 55.0 & 55.7 & 54.9 & 4.7 & 4.5 & 5.1 & 69.5 & 70.7 & 69.9 \\
\hline $\begin{array}{l}\text { Población ocupada con jornada menor a } 40 \text { horas a la } \\
\text { semana por motivos de quehaceres domésticos y } \\
\text { razones familiares (\$6) }\end{array}$ & 8.3 & 7.9 & 7.5 & 0.7 & 0.9 & 1.0 & 16.7 & 15.9 & 14.4 \\
\hline $\begin{array}{l}\text { PET que no busca trabajo por motivos de quehaceres } \\
\text { domésticos y razones familiares (\%) }\end{array}$ & 52.1 & 52.7 & 51.9 & 1.0 & 3.8 & 4.2 & 51.1 & 69.6 & 69.0 \\
\hline $\begin{array}{l}\text { Población que abandona (desocupada) su trabajo por } \\
\text { motivos de realizar tareas del hogar (\%) }\end{array}$ & 2.0 & 1.8 & 2.7 & 0.2 & 0.3 & 0.7 & 8.2 & 6.6 & 8.6 \\
\hline \multicolumn{10}{|l|}{ Oportunidades de trabajo } \\
\hline Tasa de empleo (\%) & 58.1 & 59.0 & 58.2 & 74.4 & 75.3 & 73.7 & 45.0 & 45.6 & 45.2 \\
\hline Tasa de desempleo $(\$ \%)$ & 6.3 & 5.9 & 7.3 & 8.2 & 7.5 & 9.0 & 3.7 & 3.6 & 4.9 \\
\hline Tasa de empleo juvenil (\%) & 44.2 & 50.1 & 43.4 & 59.4 & 65.8 . & 57.5 & 29.9 & 35.4 & 30.0 \\
\hline \multicolumn{10}{|l|}{ Empleo precario } \\
\hline Tasa de Subempleo (\%). & 33.5 & 31.9 & 34.5 & 31.8 & 29.5 & 33.0 & 35.6 & 34.8 & 36.4 \\
\hline Tasa de empleo informal (\%) & 47.5 & 48.6 & 50.4 & 41.1 & 43.1. & 44.6 & 55.1 & 55.3 & 57.4 \\
\hline Famillares no remunerados (\$) & 6.7 & 6.7. & 7.2 & 5.5 & 6.2 & 7.1 & 8.2 & 7.5 & 7.2 \\
\hline \multicolumn{10}{|l|}{ Segregación ocupacional } \\
\hline Segregación por sexo (Indice de Duncan) & 0.450 & 0.452 & 0.479 & & & & & & \\
\hline $\begin{array}{l}\text { Hombres/mujeres ocupadas en ramas masculinizadas } \\
\text { (\%) }\end{array}$ & & & & 50.6 & 51.8 & 54.2 & 7.6 & 8.5 & 8.5 \\
\hline $\begin{array}{l}\text { Hombres/mujeres ocupadas en ramas femenizadas } \\
\text { (\$) }\end{array}$ & & & & 8.5 & 7.6 & 7.2 & 26.6 & 26.6 & 27.3 \\
\hline \multicolumn{10}{|l|}{ Trabajo infantil } \\
\hline Tasa de trabajo infantil (\%) & 15.0 & 15.4. & 15.4 & 21.3 & 21.9 & 22.3 & 8.5 & 8.7 & 8.3 \\
\hline Tasa de niñez ocupada con edad no autorizada (\%) & 9.2 & 10.4 & 8.6 & 9.2 & 9.6 & 7.9 & 9.3 & 12.3 & 10.5 \\
\hline \multicolumn{10}{|l|}{$\begin{array}{l}\text { Ejercicio del poder y acceso a la propiedad de las } \\
\text { empresas }\end{array}$} \\
\hline Población ocupada en calidad de empleadora (\%) & 4.5 & 4.4 & 4.3 & 5.5 & 5.6 & 5.4 & 3.2 & 2.6 & 2.8 \\
\hline $\begin{array}{l}\text { Población ocupada en cargos de dirección y } \\
\text { funcionariado (\%) }\end{array}$ & 1.6 & 1.7 & 1.5 & 2.1 & 1.8 & 1.6 & 0.9 & 1.5 & 1.3 \\
\hline \multicolumn{10}{|l|}{ Salarios } \\
\hline Salarios promedios (USS) & 260.6 & 266.1 & 277.6 & 281.7 & 284.4 & 303.5 & 231.9 & 241.0 & 247.1 \\
\hline $\begin{array}{l}\text { Salarios promedios en puestos superiores de } \\
\text { dirección y funcionariado (USS) }\end{array}$ & $1,152.67$ & $1,124,64$ & 942.47 & $1,167.74$ & $1,247.62$ & 997.27 & $1,106.7$ & 916.1 & 851.8 \\
\hline $\begin{array}{l}\text { Salarios promedios de personas con } 13 \text { años y más de } \\
\text { estudios aprobados (USS) }\end{array}$ & 601.73 & 625: & 601.35 & 697.77 & 711.67 & 662.25 & 492.8 & 528.7 & 544.0 \\
\hline \multirow{2}{*}{\multicolumn{10}{|c|}{ Educación }} \\
\hline & & & & & & & & & \\
\hline Población económicamente inactiva que estudia (\%) & 19.7 & 20.4 & 21.3 & 39.2 & 41.8 & 42.3 & 14.1 & 14.1 & 14.9 \\
\hline $\begin{array}{l}\text { Población económicamente activa con } 13 \mathrm{y} \text { más años } \\
\text { de estudios aprobados (\%) }\end{array}$ & 12.8 & 13.0 & 12.9 & 11.4 & 11.5 & 10.7 & 14.7 & 15.2 & 16.1 \\
\hline \multicolumn{10}{|l|}{ Estabilidad } \\
\hline $\begin{array}{l}\text { Población ocupada que han firmado contrato de } \\
\text { trabajo (\%) }\end{array}$ & 18.5 & 19.5 & 19.2 & 20.2 & 20.6 & 21.3 & 16.5 & 18.2 & 16.9 \\
\hline Asalariado permanentes (\$6) & 40.5 & 41.2 & 37.1 & 43.1 & 44.1 & 38.9 & 37.1 & 37.4 & 34.7 \\
\hline & & & & & & & & & \\
\hline \multicolumn{10}{|l|}{ Seguridad } \\
\hline Población ocupada cubierta por el ISsS ( $\mathbf{W}$ ) & 34.8 & 33.6 & 32.3 & 32.9 & 32.9 & 31.2 & 37.7 & 34.7 & 33.9 \\
\hline Densidad de cotización (\%) & 36.0 & 31.2 & 28.5 & 35.4 & 31.2. & 28.8 & 36.9 & 31.1 & 28.1 \\
\hline Población afiliada a las AFP (X) & 72.7 & 77.4 & 82.0 & 71.1 & 73.8 & 78.1 & 74.7 & 82.2 & 87.4 \\
\hline \multicolumn{10}{|l|}{ Sindicalización } \\
\hline Tasa de sindicalización (\$) & 14.0 & 14.1 & 15.2 & 18.4 & 18.3 & 19.8 & 5.2 & 5.6 & 6.0 \\
\hline Cobertura de los contratos colectivos (\%) & 4.9 & 4.7 & 4.8 & 5.8 & 5.5 & 5.7 & 3.2 & 3.1 & 3.6 \\
\hline
\end{tabular}

Fuente: Elaboración propia a partir de EHPM, MTPS y SIP. 


\subsection{Segregación ocupacional}

Una de las repercusiones que tiene la división sexual del trabajo es que genera una segregación sexual del mercado de trabajo y/o tiende a concentrar a un mayor número de hombres o mujeres en actividades económicas en función del sexo, de tal manera que esto se traduce en desigualdades de género en el empleo y en procesos de discriminación. Por ello la Plataforma de Beijing considera necesario que los países avancen con sus compromisos de eliminar la segregación en las ocupaciones promoviendo una igual participación de las mujeres.

En cuanto a la fuerza de trabajo ocupada en actividades que por el orden de género se consideran un estamento masculino o femenino, es preciso señalar que los hombres tienden a concentrarse más en las ramas masculinizadas que las mujeres en las ramas de actividad feminizadas: el 54.2\% de los hombres están ocupados en actividades propias de estos, mientras que el porcentaje de mujeres en actividades feminizadas es del 27.3\%. Asimismo, los datos indican una baja participación de hombres y mujeres en actividades que por la socialización de género no son propias de su género: sólo el $8.5 \%$ de las mujeres están en ramas masculinizadas y un $7.2 \%$ de hombres en feminizadas.

\subsection{Ejercicio del poder y acceso a la propiedad de las empresas}

En los hogares, la reproducción y acatamiento de las prescripciones y estereotipos del orden género evidencian relaciones desiguales de poder entre hombres y mujeres, y son los hombres los que mayoritariamente ejercen la autoridad y el control sobre los recursos. La economía no está exenta de ese orden de género; el ejercicio del poder, el control de los recursos y acceso a la propiedad de las empresas es un asunto de hombres como lo señalan los indicadores: el $5.4 \%$ de los hombres ocupados trabajan en calidad de empleadores, mientras que sólo el 2.8\% de las mujeres están en dicha categoría ocupacional. Adicionalmente, la distribución de la población dentro de esta categoría constata nuevamente la mayor participación de los hombres: el 72.3\% de las personas empleadoras son hombres y el $27,7 \%$ mujeres, por lo que la propiedad de las empresas es masculinizada.

Respecto al ejercicio del poder en las empresas, las personas en cargos de dirección y funcionariado permiten una aproximación a ficha función; para 2009 los hombres ocupados en este grupo ocupacional (1.6\%) superaban en 0.3 puntos porcentuales a las mujeres dentro del mismo grupo (1.3\%); entre 2007 y 2009 la diferencia en la participación se había reducido considerablemente, pasando de 2.01 a 0.3 puntos. No obstante, del total de personas dentro de este grupo, los hombres participan con $62.1 \%$ y las mujeres con un $37.9 \%$; por lo que para lograr una participación paritaria es necesario fomentar el ascenso de las mujeres. 


\subsection{Salarios}

Las mujeres no sólo se ven discriminadas en su inserción al trabajo remunerado; una vez insertadas en la esfera productiva del mercado de trabajo se enfrentan a procesos de discriminación salarial en trabajos de igual valor y hasta en trabajos en los cuales las mujeres, según los roles de género tradicionales, poseen "cualidades innatas" para realizarlos mucho mejor que los hombres.

Según los indicadores, hay evidencia de desigualdad salarial entre hombres y mujeres, el salario promedio de las mujeres para 2009 era de US\$247.10 y US\$ 305.50 para los hombres, con una brecha salarial del $18.6 \%$, es decir, que los hombres ganan un $18.6 \%$ más que la mujeres.

En cuanto a los salarios, en uno de los grupos ocupacionales mejor remunerados y en donde el trabajo es de igual valor para ambos sexos: dirección y funcionariado, los hombres ganan $14.6 \%$ más que las mujeres.

Otro hallazgo interesante es que el trabajo doméstico en la esfera remunerada es mucho mejor valorado y remunerado si lo realiza un hombre; esto constata la permanencia del orden patriarcal que tiende a desvalorizar lo femenino. Si bien desde el orden patriarcal se supone que las mujeres tienen capacidades innatas para realizar el trabajo doméstico, esto implicaría que las mujeres tienen una ventaja comparativa con respecto a los hombres y que en la esfera del mercado debería traducirse en un mayor salario; sin embargo, los hombres que se dedican al servicio o trabajo doméstico remunerado ganan un $29.5 \%$ más que las mujeres.

\subsection{Educación o formación de la fuerza de trabajo}

De acuerdo con los indicadores, los hombres en estado de inactividad tendrían mejores oportunidades que las mujeres en la esfera productiva o de insertarse a un trabajo mejor remunerado, ya que el $42.3 \%$ de los hombres exponen como razón de su inactividad el motivo de estar estudiando, en tanto que solo el $14.9 \%$ de las mujeres son inactivas por dicho motivo.

Una de las dificultades que presenta el mercado de trabajo salvadoreño, es la poca cualificación y escolaridad de su fuerza de trabajo: sólo el $11.2 \%$ de la PET tiene trece y más años de estudios aprobados; los hombres tienden a tener una mayor participación en los rangos de escolaridad más altos, excepto en la PEA en donde el porcentaje de mujeres con 13 años de estudios es del $16.1 \%$ y de los hombres del 10.7\% (también los superan en el rango de diez a doce años).

\subsection{Estabilidad laboral}

La CEDAW establece que las mujeres tienen derecho a la estabilidad en el empleo; por otra parte, la noción de trabajo decente aboga por la protección 
social y ello implica reducir la incertidumbre de las personas en sus puestos de trabajo; la certidumbre de la permanencia en el trabajo y la existencia de mecanismo de protección contra despidos injustificados le proporciona a la fuerza de trabajo bienestar y reduce tensiones en el hogar.

La permanencia es un indicador de estabilidad laboral, por lo tanto el crecimiento de la población trabajadora en la categoría ocupacional de salariados permanentes permite una aproximación. Los datos constatan que las mujeres participan menos en comparación con los hombres como asalariadas permanentes, en 2009 sólo el $34.7 \%$ de las mujeres lo eran, mientras que el $38.9 \%$ de los hombres eran asalariados permanentes.

\section{Gráfico 1}

Estatus contractual de la población ocupada en el sector privado, 2009

(Porcentajes)

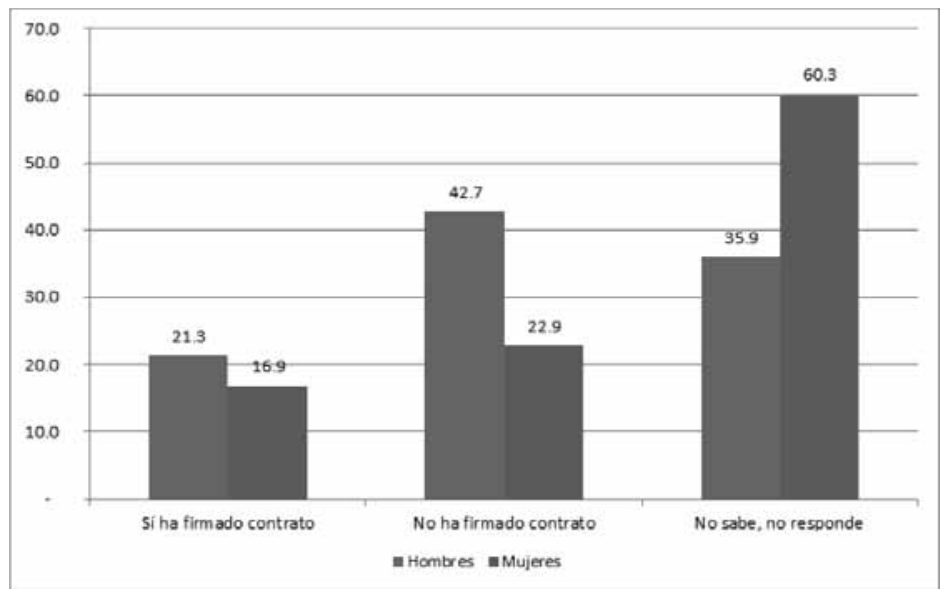

Fuente: Elaboración propia a partir de la EHPM.

Se excluye servicios domésticos y familiares no remunerados y población trabajadora en el sector agrícola por ser regímenes especiales, al igual que los ocupados en el sector público.

En otro orden, el contrato de trabajo constituye uno de los instrumentos legales que asegura la estabilidad. Con respecto a este indicador, el mercado de trabajo salvadoreño presenta bajos niveles de protección de la población trabajadora; del conjunto de ocupados en el sector privado, sólo el $19.2 \%$ tiene la certeza que ha firmado contrato de trabajo, el $33.3 \%$ no ha firmado y el $47.4 \%$ no sabe o no responde en cuanto a su estatus contractual. 
En el Gráfico 1 se observan los datos, en donde el porcentaje de hombres con contrato supera en 4.4 puntos al porcentaje de mujeres; asimismo relativamente hay más hombres que mujeres que indican que no han firmado contrato, pero las mujeres superan significativamente a los hombres en cuanto a que no tienen certeza de su estatus contractual, ya que el $60.3 \%$ de estas no sabe o no responde al respecto.

\subsection{Seguridad social}

El acceso a la seguridad social constituye otro de los derechos que desde la CEDAW debe garantizársele en iguales condiciones a mujeres y hombres; y como se señaló anteriormente el acceso a las prestaciones que ofrece la seguridad social son fundamentales para contar con una fuerza de trabajo productiva y para asegurarle un retiro digno a la población trabajadora.

La seguridad social constituye uno de los grandes déficits del mercado de trabajo salvadoreño. Los niveles de cobertura de la seguridad social son muy bajos: para 2007 el $34.8 \%$ de la población ocupada estaba cubierta por la seguridad social, para el 2009 se reduce a un $32.3 \%$ afectando a hombres y mujeres que han sufrido una reducción de la cobertura de la seguridad social, aunque la dinámica de reducción ha sido mayor en las mujeres.

En cuanto a los datos sobre las personas ocupadas que aportan al fondo de jubilaciones y pensiones, se señala que si bien el $82.2 \%$ de la población ocupada está afiliada a las administradoras de fondo de pensiones, no todas están aportando al fondo, sólo el $28.1 \%$ de las personas afiliadas cotiza efectivamente (densidad de cotización) a las administradoras de pensiones; la tendencia en los últimos años ha sido hacia la baja y la mujeres presentan una tasa de densidad levemente menor que la de los hombres.

\subsection{Trabajo infantil}

La noción del trabajo decente tiene implícita la erradicación del trabajo infantil (especialmente sus peores formas), el cual tiene sus repercusiones en términos de género y en el desarrollo y bienestar de la niñez. De acuerdo con los registros de la EHPM del total de niñez ocupada el $72.2 \%$ son niños y el $27,8 \%$ son niñas; la tasa de trabajo infantil a nivel del país es del $15.4 \%$, es decir, que el $15.4 \%$ de las niñez entre 10 y 17 años realiza una actividad laboral o está ocupada; entre 2008 y 2009 la tasa no ha variado, pero la incidencia es mayor en los niños; la tasa de trabajo infantil de los niños es de $22.3 \%$ y de las niñas es de $8.3 \%$, por lo que es un fenómeno que afecta especialmente a los niños.

\subsection{Sindicalización}

La CEDAW preocupada por erradicar la discriminación contra la mujer expone que los Estados parte garanticen a las mujeres, en iguales condiciones que 
los hombres, el derecho a participar en organizaciones y asociaciones que intervienen en la vida pública y privada del país. Por otra parte, la Plataforma de Acción establece que los países deben adoptar acciones para promover y proteger la igualdad de derecho de hombres y mujeres en la participación en actividades políticas y libertad de asociación; además, alentar a que las organizaciones sindicales consigan la igualdad de género en sus órganos de adopción de decisiones y negociación. Mientras que la noción de trabajo decente, tiene implícito el derecho fundamental de toda persona trabajadora a la libre sindicalización.

Tanto las disposiciones de la CEDAW como de la Plataforma de Acción tienen una vigencia al constatarse que la sindicalización es un espacio masculinizado: del total de personas afiliadas a los sindicatos el $86.8 \%$ son hombres y un $13.2 \%$ son mujeres. Además, los datos indican una baja cultura sindical en el mercado de trabajo salvadoreños, ya que solo el $15.2 \%$ de sus asalariados están sindicalizados y en términos de género se observan marcadas diferencias: las mujeres tienen una tasa de sindicalización del $6 \%$ y los hombres del $19.8 \%$, lo que consecuentemente puede repercutir en relaciones desiguales de poder al interior de las organizaciones y en un sesgo masculino en las reivindicaciones laborales.

\section{Conclusiones}

- Los indicadores constatan que el mercado de trabajo reproduce o se ve determinado por muchas de las prescripciones del orden patriarcal, al evidenciar la existencia de desigualdades entre mujeres y hombres en cuanto a condiciones, oportunidades y derechos laborales, muchas de las cuales están arraigas en los estereotipos, prescripciones y roles de género propios del patriarcado. No cabe duda de la vigencia de la división sexual del trabajo como factor que condiciona la inserción laboral de los hombres y mujeres salvadoreñas; y de la permanencia de los dos principios sobre lo que se sustenta en patriarcado: sobrevaloración de lo masculino y desvaloración de lo femenino.

- En términos generales, las mujeres con respecto a los hombres se encuentran levemente más pobres, mal remuneradas, con menos oportunidades en el trabajo remunerado, cargan mayoritariamente con el trabajo del hogar, están perdiendo cobertura de la seguridad social y participan más en empleos precarios. Por otra parte, los hombres tienen una mayor participación con respecto a las mujeres en las oportunidades de empleo, trabajo infantil, organización sindical, gozan de mayor estabilidad laboral, se mantienen estables en cuanto a la cobertura de la seguridad y protección social, y reciben mayores salarios. 
- La seguridad social constituye uno de los grandes déficits del mercado de trabajo, pese a que el porcentaje de hombres con acceso a la seguridad social es similar al de las mujeres. La tendencia es que cada vez hay menos población cubierta por los mecanismos de la seguridad social y son las mujeres a quienes más afecta esta dinámica; la caída del porcentaje de población cubierta por la seguridad social y de la densidad de cotización es más acentuada en las mujeres que en los hombres.

- Las áreas o componentes del mercado de trabajo en las que habrá que generar medidas de política para la igualdad son: trabajo no remunerado, sindicalización seguida por poder en el trabajo y oportunidades de empleo, así como también en materia salarial y disponibilidad de ingresos. En tanto que las áreas con mejor desempeño (o con brechas de género reducidas) en términos de igualdad son seguridad social y empleos estables, aunque el principal reto del país reside en ampliar el número de personas con acceso a la seguridad social y con mayor estabilidad en el empleo.

- No se pone en duda los esfuerzos que hasta la fecha el país ha realizado en cuanto a políticas públicas, para proporcionar a hombres y mujeres un trabajo decente en condiciones de equidad. Sin embargo, dichos esfuerzos no han sido del todo suficientes, el hecho que las mujeres se enfrenten a mayores niveles de pobreza, asuman una carga mayor del trabajo reproductivo, sufran de discriminación salarial, obtengan menores salarios, tengan una mayor incidencia en el empleo precario, menor cobertura de la seguridad social, menor estabilidad laboral y menor participación en las estructuras organizativas del mercado de trabajo evidencia que las mujeres tienen trabajos con alto déficit en términos de libertades, equidad, seguridad y dignidad humana, los cuales constituyen los principios claves de un trabajo decente.

- Los hallazgos estarían señalando la necesidad de que El Salvador haga una evaluación a conciencia del estado de cumplimiento de los compromisos en materia económica y laboral asumidos a través de la CEDAW y la Plataforma de Acción de Beijing. También, es necesaria una revisión del marco legal e institucional vigente para la igualdad de género y adelanto de las mujeres, así como de la pertinencia y efectividad de las políticas para que hombres y mujeres tengan las mismas oportunidades y condiciones de trabajo. 


\section{Bibliografía}

J. Butler, El género en disputa: el feminismo y la subversión de la identidad. Editorial Paidos Mexicana, 2001

CEPAL-UNFPA. Masculinidad y factores socioculturales asociados al comportamiento de los hombres: estudio en cuatro países de Centroamérica. UNFPA-CEPAL, Nicaragua, 2005

DIGESTYC. Encuesta de Hogares de Propósitos Múltiples. Dirección de Estadísticas y Censos de El Salvador. Ministerio de Economía. El Salvador, 2009.

DIGESTYC. Índice de Precios al Consumidor de El Salvador. Serie 5, No. 12. Dirección de Estadísticas y Censos de El Salvador. Ministerio de Economía. El Salvador, 2009a

B. Miedes: "Principales condicionantes de la desigualdad de género en el mercado laboral: aspectos reproductivos". Resumen de la unidad No. 3 del módulo Desigualdades de Género en los Mercados de Trabajo. Universidad de Huelva, 2010

Naciones Unidad. Declaración y Plataforma de Acción de Beijing. Cuarta Conferencia Mundial sobre la Mujer. Naciones Unidas, 1995

Naciones Unidad. Convención sobre la Eliminación de Todas las Formas de Discriminación Contra la Mujer. Naciones Unidas. http://www.un.org/ womenwatch/daw/cedaw/text/sconvention.htm 1979, Consultado el 16 de noviembre de 2008.

OIT). Memoria del Director General: Trabajo Decente. Conferencia Internacional del Trabajo, 87. ${ }^{a}$ reunión. Oficina Internacional del Trabajo. http:// www.ilo.org/public/spanish/standards/relm/ilc/ilc87/rep-i.htm 1999, Consultado el 20 de diciembre de 2009.

OIT. Informe VI: La igualdad de género como eje del trabajo decente. Conferencia Internacional del Trabajo, 98. ${ }^{\text {a }}$ reunión. Oficina Internacional del Trabajo. Ginebra, 2009.

OIT-IPEC. Explotación sexual comercial y masculinidad: un estudio regional cualitativo con hombres de la población general. OIT-IPEC. Costa Rica, 2004

Picchio, Antonella "Un enfoque macroeconómico ampliado de las condiciones de vida” en Carrasco, C. (ed.) (2001). Tiempos, trabajos y género, Universitat de Barcelona, Barcelona, 2001 
M. A. Rivas. "Desigualdades de Género en El Mercado Laboral: un Problema Actual”. Departament d'Economia Aplicada. Universitat de les Illes Balears. $\quad$ www.uib.es/depart/deaweb/deawp/pdf/w6.pdf 2004. Consultado el 7 de mayo de 2010.

J. Scott. “El género: una categoría útil para el análisis histórico”. En Historia y género: las mujeres en la Europa moderna y contemporánea, James y Amelang y Mary Nash (eds.), Edicions Alfons el Magnanim, Institució Valencina d Estudis i Investigació. Este artículo fue publicado en Ingles como "Gender: A Useful Category of Historical Analysis" en American Historical review, 91,1986, pp. 1053-1075, 1990. 